\title{
Neutrino oscillations and flavor theories
}

\author{
José W. F. Valle * \\ AHEP Group, Institut de Física Corpuscular - C.S.I.C. \\ Universitat de València, Parc Cientific de Paterna. \\ C/Catedratico José Beltrán, 2 E-46980 Paterna (València) - SPAIN \\ E-mail: valledific.uv.es
}

I discuss neutrino mixing ansatze, such as the generalized Tri-bimaximal and bi-large mixing patterns, and their utility in describing neutrino oscillation data. Unitarity tests and probes of the absolute neutrino mass scale are briefly discussed. A short overview of neutrino mass generation is given, including an orbifold approach to the flavor problem and the resulting implications, e.g. the golden quark-lepton mass relation, $0 v \beta \beta$ and neutrino oscillation predictions.

Proceedings of the 40th International Conference on High Energy Physics (ICHEP2020) July 28 - August 6, 2020, Prague, Czech Republic

${ }^{*}$ This work was supported by the Spanish grants FPA2017-85216-P (AEI/FEDER, UE), PROMETEO/2018/165 (Generalitat Valenciana) and the Spanish Red Consolider MultiDark FPA2017-90566-REDC. 
Neutrino mixing Here I start with the interpretation of the neutrino oscillation results reported by Tortola. A striking feature of the observed pattern of neutrino mixing, is that it is characterized by two large angles, one nearly maximal and the other nearly thirty degrees. A simple way to account for this is the TBM ansatz. Although it captures the most salient features of the oscillation phenomenon, TBM is now in conflict with the non-zero value of $\theta_{13}$ indicated by reactor experiments [1]. Fortunately, there are systematic ways of generalizing patterns with manifest $\mu-\tau$ reflection symmetry, such as TBM. By exploiting partially conserved generalized CP symmetries $[2,3,4]$ one can develop "revamped" TBM patterns. This way one obtains two-parameter mixing schemes which can be not only viable, but also predictive [5]. As an example, we present one in which the CP phase is predicted to lie on the green band in Fig. 1, left [6]. Clearly the overlap of the predicted band with the 3 -neutrino global oscillation region covers a narrow $\delta_{C P}$ range ${ }^{1}$.
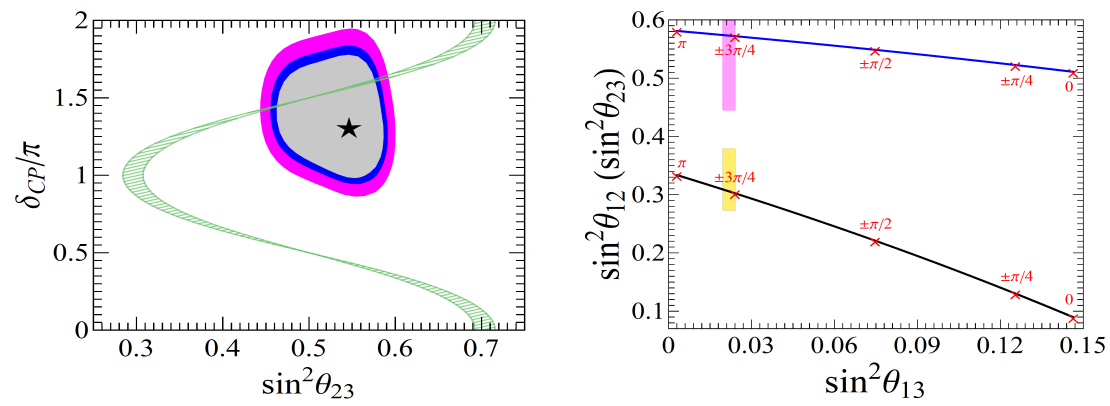

Figure 1: Left: Predicting the CP phase in a realistic generalized TBM scenario, from [6]. Right: Predicting solar and atmospheric mixing angles within the "bi-large" scheme of Ref. [7].

Bi-large neutrino mixing $[8,9]$ builds up on the observation that the Cabibbo angle is similar in magnitude to the reactor mixing angle and may be used to seed flavor mixing. One finds that the good measurement of $\theta_{13}$ at reactors allows us to predict both solar and atmospheric mixing angles [7] quite acurately, as seen in the right panel of Fig. 1. It also leads to sharp predictions for the modulus of the CP phase. There are also "softer" bi-large-type lepton mixing schemes in which the oscillation parameters are given in terms of two free parameters [10].

Unitarity tests If light neutrinos acquire their masses from the exchange of heavy neutral singlet mediators, as in the simplest seesaw mechanism, then their propagation will be described effectively by a non-unitary mixing matrix $[11,12,13,14,15]$. Most generally, this is expressed as

$$
\left(\begin{array}{ccc}
\alpha_{11} & 0 & 0 \\
\alpha_{21} & \alpha_{22} & 0 \\
\alpha_{31} & \alpha_{32} & \alpha_{33}
\end{array}\right) U,
$$

where the unitary matrix $U$ is pre-multiplied by a triangular matrix $[11,14,15]$ whose diagonal entries are real and less than unity, while the off-diagonal ones are small but complex. Due to an intrinsic degeneracy between $\delta_{C P}$ and the phase in $\alpha_{21}$ [16] it so happens that the latter plays a crucial role in the $\mathrm{CP}$ measurement. This confusion problem can be mitigated [17] by having better limits on the magnitude of $\alpha_{21}$. Current bounds are at the $\%$ level, see Ref. [18]. The short-baseline neutrino program at Fermilab is ideally suited to probe the unitarity of the lepton mixing matrix,

\footnotetext{
${ }^{1}$ This scheme also promotes the precise measurement of $\theta_{13}$ into predictions for the solar angle.
} 
with potentially better sensitivities [19]. Apart from their motivation as a seesaw probe, unitarity tests at short distance setups are required for a robust $\mathrm{CP}$ measurement in the far detector.

Absolute neutrino mass scale Beta decay endpoint studies and cosmological observations probe the absolute neutrino mass, inaccessible to oscillations. For example, the Katrin experiment has set an upper limit of $1.1 \mathrm{eV}$ (at 90\% C.L.) [20], which holds irrespective of whether neutrinos are Dirac or Majorana. In the latter case there is also a neutrinoless $0 v \beta \beta$ variety of double beta decay mediated by the virtual Majorana neutrinos. In Fig. 2, left panel from Ref. [21], we display the $0 v \beta \beta$ amplitute versus the degeneracy parameter $\eta$, so that $\eta \simeq 1$ corresponds to nearly degenerate neutrinos. The normal-ordered neutrino region is indicated in the left blue band, while inverted ordering gives the upper-right branch. Thanks to the Majorana phases [22], the $0 v \beta \beta$ amplitude can vanish for normal ordering ${ }^{2}$. The vertical band is excluded by cosmological observations [21], while the horizontal ones denote the current limit and the future expected sensitivities.
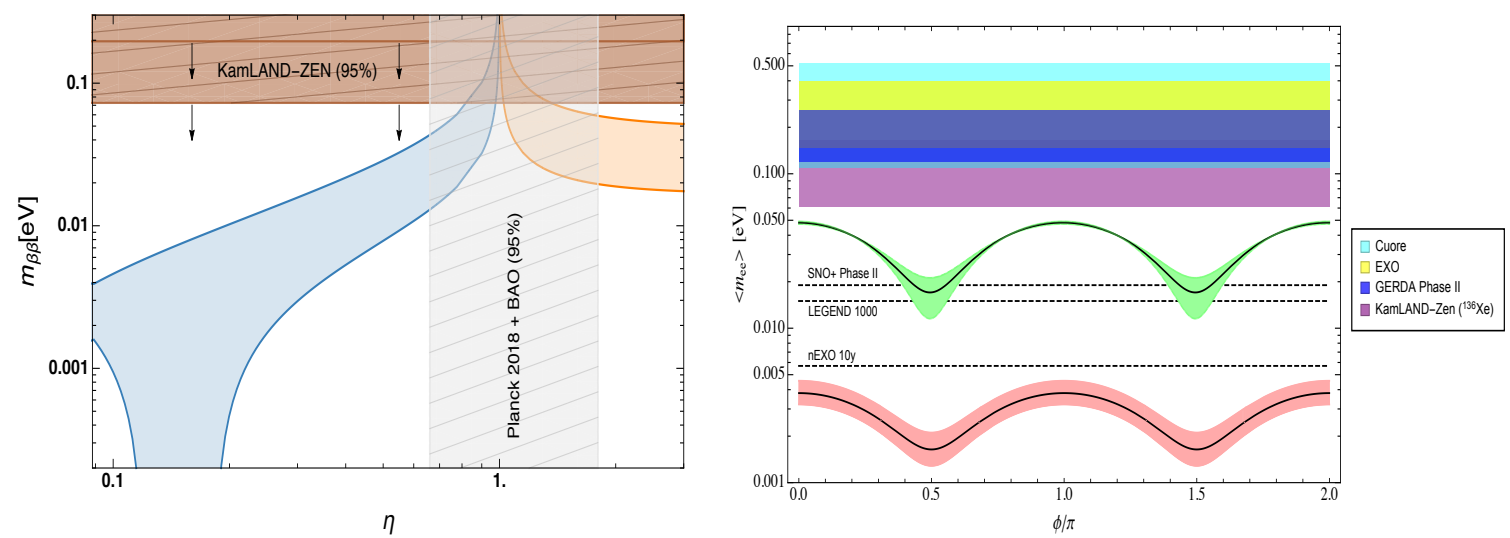

Figure 2: $0 v \beta \beta$ decay amplitude in a generic three-neutrino scheme (left) and in a two-massive-neutrinoscheme [26, 27], right. The vertical nearly-degenerate band is excluded by cosmology [21].

Note that if one of the three neutrinos is massless or nearly so, as in the "missing partner" seesaw [22], no cancellation is possible, even for normal-ordering [26, 27]. The resulting regions correlate with the only free parameter available, i.e. the relative neutrino Majorana phase, as seen in Fig. 2, right panel. There is a lower bound, though it lies below detectability in upcoming experiments. In contrast, the lower bound found for inverse ordering lies higher than in the generic three-neutrino case, giving hope that the coming experiments [28] may not only prove the Majorana nature by the black-box theorem [29], but also determine the relevant Majorana phase.

Origin of neutrino mass Even though the Standard Model lacks neutrino masses, these can arise from a unique dimension-five operator characterizing lepton number non-conservation [30]. Seesaw completions open the way for a dynamical understanding of small neutrino masses. The mediators, "right-handed" singlet neutrinos in type-I, or the scalar triplet in type-II, were originally thought to lie at a high mass scale, associated to SO10 unification. In addition to the standard vacuum expectation value (vev) responsible for electroweak breaking, there are new "neutrinophilic" vevs. The most general seesaw description of neutrino mass generation is obtained by adopting the minimal $\mathrm{SU}(3)_{\mathrm{c}} \otimes \mathrm{SU}(2)_{\mathrm{L}} \otimes \mathrm{U}(1)_{\mathrm{Y}}$ gauge group [22], instead of left-right symmetric extensions.

\footnotetext{
${ }^{2}$ Note however that in many models there is a $0 v \beta \beta$ lower bound even for normal ordering, since the cancellation is prevented by the flavor structure of the leptonic weak interaction vertex [23, 24, 25]. However this is model-dependent.
} 
The new vevs drive the spontaneous violation of lepton number, accompanied by a Goldstone boson, dubbed Majoron [31, 32]. Such dynamical 3-2-1 seesaw mechanism can improve the consistency properties of the electroweak vacuum, such as stability [33, 34, 35].

In such general seesaw one can have any number of "right-handed" neutrino mediators, since they are gauge singlets. As an example, one may consider a seesaw scheme with only two "right" neutrinos, implying that one of the "left" neutrinos remains massless [22]. Likewise, a "missing partner" seesaw scheme with just one singlet mediator leads to two massless neutrinos. A "dark sector" can provide the other (solar) neutrino scale in a "calculable" way, as in the scoto-seesaw [36]. The lower bound on $0 v \beta \beta$ decay shown in Fig. 2 (right panel) holds in both missing-partner cases.

Conversely, one may add more "right-" than "left-handed" neutrinos, for example, one can have two isosinglets per family, sequentially. By imposing lepton number conservation on such scheme one finds that light neutrinos are massless, exactly as in the Standard Model, as long as lepton number symmetry is exact. In contrast to the Standard Model case, however, lepton flavor is violated, and similarly, CP symmetry. This has two important implications. On the one hand, it elucidates the meaning of flavor and $\mathrm{CP}$ violation in the leptonic weak interaction, implying that such processes need not be suppressed by the small neutrino masses, and hence can be large. This construction also provides a template for building genuine "low-scale" realizations of the seesaw mechanism by adding a small lepton number violation seed [37, 38, 39, 40]. In contrast to the high-scale seesaw mechanism, there is a rich phenomenology in low-scale seesaw, including nonunitarity effects in neutrino propagation, large lepton flavor violation rates and the possibility of having the neutrino mass mediators accessible to collider energies. For a discussion and original references see Chapter 15 in [41].

Apart from inessential technicalities, the basic seesaw philosophy also works for the case of Dirac neutrinos. One has both type-I and type-II Dirac seesaw realizations, and the small neutrino masses are also symmetry-protected. Notice that there might be a deep reason for neutrinos to be Dirac-type. For example, this could signal the stability of dark matter $[42,43]$ or the existence of an underlying Peccei-Quinn symmetry [44].

Neutrinos and the flavor and dark matter problems The Standard Model lacks an organizing principle in terms of which to understand the replication of fermion families, their masses and mixings. Here lies one of the deepest problems in our field. The discovery of oscillations have left an important legacy in particle physics, by establishing that (i) neutrino masses exist and (ii) leptons mix differenty from quarks, with two large mixing angles. Understanding this from first principles constitutes a formidable challenge. In trying to account for the observed neutrino mixing pattern within UV-complete 3-2-1 family symmetry models one discovers, perhaps, one of the ingredients towards the complete theory, namely the "golden quark-lepton mass relation" [45]

$$
\frac{m_{\tau}}{\sqrt{m_{\mu} m_{e}}} \approx \frac{m_{b}}{\sqrt{m_{s} m_{d}}} \text {. }
$$

Such relation resembles b-tau unification, except for the lack of an underlying SU(5) gauge group. Moreover, it is a genuine flavor prediction involving all of the three families. It emerges as a common prediction of many family-symmetry models [25, 45, 46, 47, 48, 49, 50, 51, 52].

There are many ways to model flavor within UV-complete schemes, and I will refrain from entering into this arcane subject. Instead, I will convey the radical idea that perhaps extra dimen- 
sions provide an adequate arena for pursuing a theory of flavor. As an example, I discuss a recently proposed theory for fermion masses and mixings in which an A4 family symmetry arises naturally from a six-dimensional spacetime after orbifold compactification. As seen in the left panel in Fig. 3 the flavor symmetry leads to the successful "golden" quark-lepton unification formula. One sees the resulting prediction for the down- and strange-quark masses at the $M_{Z}$ scale. The cyan contours represent the 1,2 and $3 \sigma$ allowed regions from the golden relation. The yellow contours show the 1,2 and $3 \sigma$ ranges of the measured quark masses at the $M_{Z}$ scale [53]. The blue region is the region consistent at $3 \sigma$ with the model's global flavor fit. The red (black) cross indicates the location of the best fit point for two model variants, MI (MII).

In the simplest "constrained" realization MI we assume that the Higgs vevs are real. Assuming real Yukawa couplings, this implies that the only source of $\mathrm{CP}$ violation is numerically fixed. This leads to a very strong predictivity for $\mathrm{CP}$ violation, including even the sign of the relevant $\mathrm{CP}$ phase. CP violation of quarks and leptons has a common source and this leads to some tension in the global fit of the flavor observables, see [51] for details. The effective neutrinoless double-beta decay mass parameter is also sharply predicted, as seen in the right panel in Fig. 3.
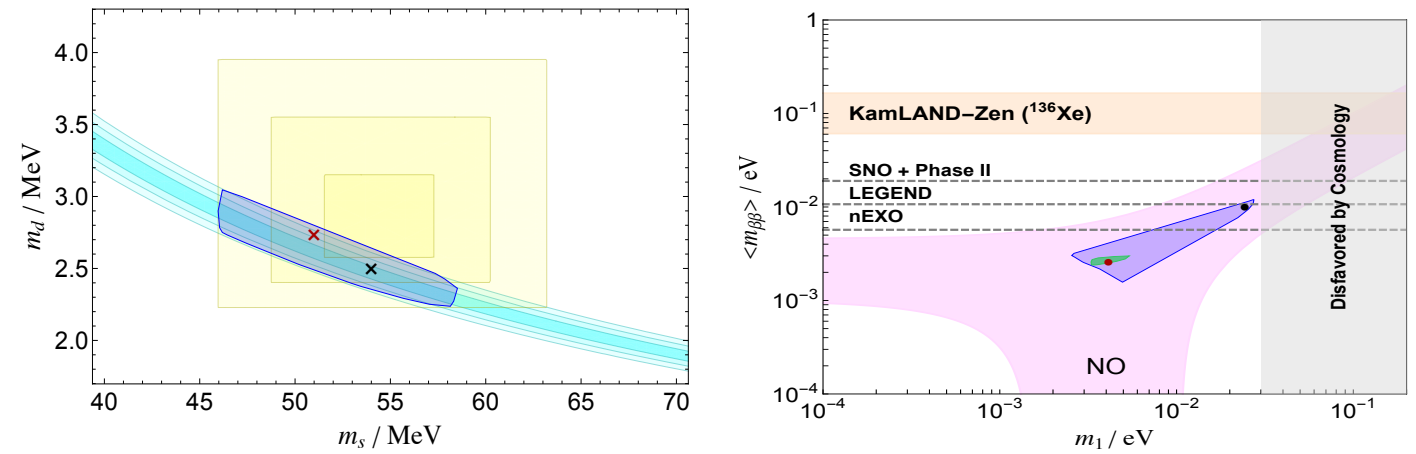

Figure 3: Predictions of the "golden" quark-lepton unification formula (left), and for the $0 v \beta \beta$ decay amplitude for MI (MII) scenarios, right. From [51], see text.

In the second model MII we relax the assumption that all the Higgs vevs are real, keeping real Yukawa couplings. In this case the tension is resolved and the fit quality is excelent, with good predictions for $0 v \beta \beta$ and neutrino oscillations. Upcoming experiments DUNE as well as LEGEND and $\mathrm{nEXO}$ offer good chances of exploring a substantial region of neutrino parameters.

To conclude, let me also comment that dark matter and neutrinos could be closely related. Out of the many ways to implement this connection, we have the so-called scotogenic approach, in which WIMP dark matter acts as the mediator of neutrino mass generation [54], and which can also be neatly realized within the above orbifold scenario, see Ref. [52] for details.

\section{References}

[1] F.P. An et al. Observation of electron-antineutrino disappearance at Daya Bay. Phys.Rev.Lett., 108:171803, 2012.

[2] Chen etal, Generalized $\mu \tau$ reflection symmetry and leptonic CP violation. Phys.Lett, B753:644, 2016

[3] Chen et al, Classifying CP transformations according to texture zeros Phys.Rev D94:033002, 2016

[4] Peng Chen, Salvador Centelles Chuliá, Gui-Jun Ding, Rahul Srivastava, and José W.F. Valle. Neutrino Predictions from Generalized CP Symmetries of Charged Leptons. JHEP, 1807:077, 2018. 
[5] Newton Nath, Rahul Srivastava, and José W.F. Valle. Testing generalized CP symmetries with precision studies at DUNE. Phys.Rev., D99:075005, 2019.

[6] Peng Chen et al, Realistic tribimaximal neutrino mixing. Phys.Rev., D98:055019, 2018.

[7] Chen et al, Predicting neutrino oscillations with "bi-large" lepton mixing Phys.Lett, B792:461, 2019

[8] S.M. Boucenna et al, Bi-large neutrino mixing and the Cabibbo angle. Phys.Rev., D86:051301, 2012.

[9] Gui-Jun Ding, S. Morisi, and J.W.F. Valle. Bilarge neutrino mixing and Abelian flavor symmetry. Phys.Rev., D87:053013, 2013.

[10] Roy et al, The Cabibbo angle as a universal seed for quark and lepton mixing Phys.Lett, B748:1, 2015

[11] J.W.F. Valle. Resonant Oscillations of Massless Neutrinos in Matter. Phys.Lett., B199:432-436, 1987.

[12] Nunokawa et al, Resonant conversion of massless neutrinos in supernovae. Phys.Rev, D54:4356, 1996

[13] S. Antusch, C. Biggio, E. Fernandez-Martinez, M.B. Gavela, and J. Lopez-Pavon. Unitarity of the Leptonic Mixing Matrix. JHEP, 0610:084, 2006.

[14] F.J. Escrihuela, D.V. Forero, O.G. Miranda, M. Tortola, and J.W.F. Valle. On the description of nonunitary neutrino mixing. Phys.Rev., D92:053009, 2015.

[15] O.G. Miranda and J.W.F. Valle. Neutrino oscillations and the seesaw origin of neutrino mass. Nucl.Phys., B908:436-455, 2016.

[16] O.G. Miranda, M. Tortola, and J.W.F. Valle. New ambiguity in probing CP violation in neutrino oscillations. Phys.Rev.Lett., 117:061804, 2016.

[17] Shao-Feng Ge, Pedro Pasquini, M. Tortola, and J.W.F. Valle. Measuring the leptonic CP phase in neutrino oscillations with nonunitary mixing. Phys.Rev., D95:033005, 2017.

[18] F.J. Escrihuela et al, Probing CP violation with non-unitary mixing in long-baseline neutrino oscillation experiments: DUNE as a case study. New J.Phys., 19:093005, 2017.

[19] O.Miranda et al, Exploring the Potential of Short-Baseline Physics Phys.Rev D97:095026, 2018

[20] M. Aker et al. An improved upper limit on the neutrino mass from a direct kinematic method by KATRIN. Phys. Rev. Lett., 123(22):221802, 2019.

[21] Lattanzi et al, Cornering (quasi) degenerate neutrinos with cosmology. JHEP, 2010:213, 2020.

[22] J. Schechter and J.W.F. Valle. Neutrino Masses in SU(2) x U(1) Theories. Phys.Rev., D22:2227, 1980.

[23] L. Dorame et al, Constraining Neutrinoless Double Beta Decay. Nucl.Phys., B861:259-270, 2012.

[24] L. Dorame et al, A new neutrino mass sum rule from inverse seesaw. Phys.Rev., D86:056001, 2012.

[25] S.F. King, S. Morisi, E. Peinado, and J.W.F. Valle. Quark-Lepton Mass Relation in a Realistic $A_{4}$ Extension of the Standard Model. Phys.Lett., B724:68-72, 2013.

[26] M. Reig et al, Bound-state dark matter with Majorana neutrinos. Phys.Lett., B790:303-307, 2019.

[27] Ivania M. Ávila et al, Minimalistic scotogenic scalar dark matter. Eur. Phys. J. C, 80(10):908, 2020.

[28] M. Agostini et al. Probing Majorana neutrinos with double- $\beta$ decay. Science, 365:1445, 2019.

[29] J. Schechter and J.W.F. Valle. Neutrinoless Double beta Decay in SU(2) x U(1) Theories. Phys.Rev., D25:2951, 1982.

[30] Steven Weinberg. Baryon and Lepton Nonconserving Processes. Phys.Rev.Lett., 43:1566-1570, 1979. 
[31] Y. Chikashige, Rabindra N. Mohapatra, and R.D. Peccei. Are There Real Goldstone Bosons Associated with Broken Lepton Number? Phys.Lett., B98:265-268, 1981.

[32] J. Schechter and J.W.F. Valle. Neutrino Decay and Spontaneous Violation of Lepton Number. Phys.Rev., D25:774, 1982.

[33] Cesar Bonilla, Renato M. Fonseca, and José W. F. Valle. Consistency of the triplet seesaw model revisited. Phys.Rev., D92:075028, 2015.

[34] Cesar Bonilla, Renato M. Fonseca, and José W. F. Valle. Vacuum stability with spontaneous violation of lepton number. Phys.Lett., B756:345-349, 2016.

[35] Sanjoy Mandal, Rahul Srivastava, and José W.F. Valle. Consistency of the minimal high-scale type-I seesaw mechanism. Phys. Rev. D, 101(11):115030, 2020.

[36] Nicolás Rojas, Rahul Srivastava, and José W.F. Valle. Simplest Scoto-Seesaw Mechanism. Phys.Lett., B789:132-136, 2019.

[37] R.N. Mohapatra and J.W.F. Valle. Neutrino Mass and Baryon Number Nonconservation in Superstring Models. Phys.Rev., D34:1642, 1986.

[38] E. Akhmedov et al, Dynamical left-right symmetry breaking. Phys.Rev., D53:2752-2780, 1996.

[39] E. Akhmedov et al, Left-right symmetry breaking in NJL approach. Phys.Lett., B368:270, 1996.

[40] Michal Malinsky, J.C. Romao, and J.W.F. Valle. Novel supersymmetric SO(10) seesaw mechanism. Phys.Rev.Lett., 95:161801, 2005.

[41] Jose W.F. Valle and Jorge C. Romao. Textbook Neutrinos in high energy and astroparticle physics. https://www.amazon.es/Neutrinos-High-Energy-Astroparticle-Physics/dp/3527411976

[42] Centelles Chuliá et al, Dirac Neutrinos and Dark Matter Stability Phys.Lett, B767:209, 2017

[43] Salvador Centelles Chuliá, Rahul Srivastava, and José W. F. Valle. Generalized Bottom-Tau unification, neutrino oscillations and dark matter Phys.Lett., B773:26-33, 2017.

[44] Alex G. Dias et al, Reloading the axion in a 3-3-1 setup. Phys.Lett., B810:135829, 2020.

[45] S. Morisi et al, Relating quarks and leptons without grand-unification. Phys.Rev., D84:036003, 2011.

[46] S. Morisi et al, Quark-Lepton Mass Relation and CKM mixing in an A4 Extension of the Minimal Supersymmetric Standard Model. Phys.Rev., D88:036001, 2013.

[47] Cesar Bonilla, Stefano Morisi, Eduardo Peinado, and Jose W.F. Valle. Relating quarks and leptons with the $T_{7}$ flavour group. Phys.Lett., B742:99-106, 2015.

[48] C. Bonilla et al, Flavour-symmetric type-II Dirac neutrino seesaw Phys.Lett, B779:257, 2018

[49] Mario Reig, José W.F. Valle, and Frank Wilczek. SO(3) family symmetry and axions. Phys.Rev., D98:095008, 2018.

[50] Francisco J. de Anda, José W.F. Valle, and Carlos A. Vaquera-Araujo. Flavour and CP predictions from orbifold compactification. Phys.Lett., B801:135195, 2020.

[51] de Anda et al, Probing the predictions of an orbifold theory of flavor. Phys.Rev., D101:116012, 2020.

[52] Francisco J. de Anda, Ignatios Antoniadis, José W.F. Valle, and Carlos A. Vaquera-Araujo. Scotogenic dark matter in an orbifold theory of flavor. JHEP, 2010:190, 2020.

[53] Stefan Antusch and Vinzenz Maurer. Running quark and lepton parameters at various scales. JHEP, 1311:115, 2013.

[54] Ernest Ma. Verifiable radiative seesaw mechanism of neutrino mass and dark matter. Phys.Rev., D73:077301, 2006. 